\title{
The Impact of a Switch from Conventional Media to Digital Media in the Treatment of Vicarious Trauma During Art Therapy
}

\author{
Elizabeth Donahue ${ }^{1}$ (D) https://orcid.org/0000-0002-6660-8022 \\ Cass Dykeman ${ }^{2}$ (D) https://orcid.org/0000-0001-7708-1409 \\ ${ }^{1}$ Antioch University Seattle \\ ${ }^{2}$ Oregon State University
}

This is the authors' preprint of a manuscript for consideration by a peer reviewed journal. Any comments are invited and can be directed to the first author at edonahue@antioch.edu

\begin{abstract}
In the United States, the use of digital media has become omnipresent and has changed how people communicate, conduct business, and create art and music. However, art therapy lags behind the current culture of prolific use of digital media. To date, there is very little experimental research on the use of digital media in the field of art therapy. The deficiency of evidence that digital media is as effective as conventional media is an obstacle to a full embrace and adoption of technology in practice by the field. The present study was designed to address this lack of evidence. Specifically, this study examined the impact of a switch from conventional to digital media in a mandala-based art therapy upon three dependent variables of interest. These variables were anxiety, vicarious traumatization, and client treatment satisfaction. An ABAB reversal design was used. Results suggested that such a switch had no impact.

Keywords: art therapy, digital media, mandala, tablet, vicarious trauma
\end{abstract}

\section{Introduction}

As technology and the internet continue to transform our culture, people are finding their lives are being influenced in ever increasing ways. In the past ten years, the proliferation of digital communication and design known as The Digital Revolution has reshaped all aspects of art, music, literature, an economics. For example, from 2000 to 2016, internet use in the US went from $52 \%$ to $88 \%$ (Pew Research Center, 2017). People are now purchasing books, movies and music, interacting with friends and family, doing personal banking, and even grocery shopping from their homes using the internet. Highly relevant to art therapists, smartphone and tablet use in the US has increased markedly. Among adults, the smartphone ownership stands at $68 \%$ and tablet ownership at $45 \%$ (Pew Research Center, 2015). Art making is also influenced by this rapid advancement of technology. While artists will always use conventional media such as canvas, paints, and markers, they are also increasingly using digital media in the form of computers, digital cameras and tablets. 
Art therapy research has yet to address the influences that digital technology has on art making in the general population. This dearth concerning digital is an obstacle to the adoption of technology in practice by the field of art therapy. Without evidence that demonstrates that art therapy with digital media is effective, the gap between art therapists and the wider culture will continue to grow.

With the aforementioned gap in mind, research literature relevant to a study of technology and art therapy was examined. Six themes emerged in this review. These themes were: (1) the efficacy of art therapy with conventional media in anxiety and vicarious traumatization, (2) the presence of anxiety and vicarious traumatization in preservice therapists, (3) the relationship between client satisfaction and art therapy outcomes (4) the gradual shift in the field of art therapy toward the use of digital media in practice, (5) the relationship of the cultural relevancy of art therapy and the use of digital media, and (6) the relationship of the efficacy of digital media and adoption of this media in art therapists. This introduction section concludes with a statement of the research question.

Art therapy with conventional media has been well-established as an effective method to treat a variety of mental health issues. Slegelis (1987) conducted an early study on mandala-making and affect. He found that participants instructed to draw inside a circle displayed more positive affect as compared to those directed to draw inside a square. Curry and Kasser (2005) examined anxiety levels in undergraduate students, as measured by the State Anxiety Inventory (Goolkasian, n.d.). In this study, subjects colored one of three provided interventions for 20 minutes: a pre-drawn mandala, a plaid design, or a free drawing. Curry and Kasser (2005) found that coloring a pre-drawn mandala or a plaid design resulted in decreased symptoms of anxiety in participants.

Though there is no current literature on the efficacy of art therapy on the treatment of symptoms of vicarious trauma, some research has shown that art therapy is effective in the treatment of PTSD. Henderson, Rosen, and Mascaro (2007) looked at undergraduate students who reported moderate symptoms of Posttraumatic Stress Disorder (PTSD) as measured by the Posttraumatic Stress Disorder Scale (PDS; Foa, 1998). Participants were randomized into two groups, one group was asked to draw an object of their choosing, and the other was asked to draw a mandala. The groups drew on three successive days. One month later, the group that created mandalas reported significantly decreased symptoms of PTSD as compared to the group that did not draw mandalas. Wilson and Lindy (1994) propose that vicarious traumatization is a form of PTSD. Art therapy may therefore also be effective in the treatment of vicarious trauma.

There is evidence that practicing therapists who work with clients who have experienced trauma are more likely to experience symptoms of anxiety, avoidance, intrusive thoughts, and other symptoms associated with vicarious traumatization. Adams and Riggs (2008) studied 129 clinical and counseling psychology graduate students across the United States who were working with clients who had experienced trauma. The researchers administered a revised Trauma Symptom Inventory (TSI, 
Briere, 1995) which measured symptoms of vicarious trauma and found that $31 \%$ of participants received scores on at least one of the TSI, indicating the presence of symptoms of vicarious trauma. Pearlman and Mac Ian (1995) administered questionnaires to 188 self-identified trauma therapists and found that those who were newer to practicing therapy were more likely to experience distressing psychological symptoms as measured by the Symptom Checklist-90-Revised (SCL-90-R, Derogatis, 1994) including symptoms associated with vicarious traumatization.

The literature shows that clients who participate in art therapy report high satisfaction with their experience. Ferszt, Hays, DeFedele, and Horn (2004) conducted an art therapy group of eight incarcerated women who had experienced the death of a loved one. The women took part in eight weekly one-hour art therapy sessions. At the conclusion of the study, seven of the eight women reported that art therapy was effective at helping them process their grief and recommended the program be continued and expanded.Gersch and Gonclaves (2010) conducted group art therapy in a school setting with 10-year-old children experiencing grief. The group met weekly for one year. At the conclusion of the group, every child reported that art therapy specifically helped them to cope with their feelings. A study of lupus patients (Nowicka-Sauer, 2007) who participated in an art therapy group focused on processing feelings related to their diagnosis revealed that these patients were satisfied with the outcome of their participation in the group. The women were asked to "draw their diseases" in a group therapy setting. At the conclusion of the group, all women reported that they believed that drawing in session was superior to traditional talk therapy.

There has been a gradual shift in art therapy toward incorporating digital media in practice. Digital media in art therapy practice may include the use of digital cameras, computers, digital drawing pads such as the Wacom Tablet, digital styluses, and other tablet computers such as the Apple iPad or the Microsoft Surface. The American Art Therapy Association (AATA) formed the AATA Technology Committee in 2011, with an intention to "gather, formulate and disseminate knowledge relevant to the use of digital technology as a therapeutic medium" and to promote the "safe and ethical use of technology in art therapy" (AATA, 2011, p. 2). The mid-to-late 2000s saw an everincreasing number of conversations about digital art therapy on blogs and art therapy forums. The Technology Committee was formed partly because of these conversations in order to further investigate the influence of digital technology on the field of art therapy. In a survey of 177 practicing art therapists, Orr (2009) found that while $88 \%$ of art therapists use technology in their practice (such as computers, printers, and accessing the internet), only $19 \%$ use technology (such as digital cameras or tablets) when working with clients. Participants who did not incorporate digital media in their practice cited several reasons for not doing so. Some reported limited time for use and training in digital media, others stated that unfamiliarity made them feel uncomfortable with the medium, and others refer to the high cost of gaining technology such as digital tablets and styluses (Orr, 2009). Further, Orr (2009) concluded that a major barrier to art therapists incorporating digital media into their practice was lack of training, and advocated that increased training opportunities for practicing art therapist be provided. 
Lastly, he also suggested that art therapy training programs should integrate education in using digital media into their curriculum.

The incorporation of digital media into the field of art therapy is essential (Orr, 2009). The field risks losing relevance and credibility in a culture that is suffused with technology if digital media is not incorporated into practice of art therapy (Kapitan, 2009). Moreover, Kapitan (2011) argues that art therapists are not including digital media at an adequate rate to meet the demands of modern clients. Therefore, art therapy lags behind the current culture of prolific use of digital media in the United States. Although many art therapists embrace the use of new technologies in their practice, many others are uncertain and tentative, and resist the use of digital media in their work (Orr, 2009). There has been inadequate research on the value and efficacy of digital media in art therapy and this dearth in the literature has resulted in "ambivalence and anxiety concerning the power of technology" (Kapitan, 2011, p. 50) to flourish in the field of art therapy.

In the event that it can be established that the use of digital media is as effective as conventional media, art therapists may be more likely to integrate it into their clinical practice. Reports of the bourgeoning efforts to add digital media to art-making with clients are mixed. Peterson (2010) surveyed 136 practicing art therapists to learn more about how they include digital media into their practice. Peterson used Rogers (2003) adoption process for media innovation to explore how art therapists adopt new technology. She found that art therapists are likely to adopt new technology when they see it as an improvement upon old technology. For example, art therapists adopted Polaroid cameras for phototherapy because pictures were ready for use within minutes rather than the days it would take to send film out to be developed (Wolf, 2007). While art therapists are adopting digital media, the rate at which they are doing so as compared to the general population is remarkably slow. Reasons for this slow rate have been attributed to the field's regard for traditional media as more therapeutic, lack of preparation and training in using new technology, a belief that the sensory experience offered by conventional media is more effective than the "cold" sensory experience offered by digital media, and inadequate access to technology due to the high cost of procurement (Carlton, 2014). The deficiency of evidence that digital media is as effective as conventional media is an obstacle to a full embrace and adoption of the technology the field of art therapy.

There were three research questions which guided this study. The first question was: In reference to GAD symptom level, what is the impact of a switch to digital media for mandala-making in art therapy for clients? The second question was: In reference to symptoms of vicarious trauma what is the impact of a switch to digital media for mandala art therapy for clients? The third question was: In reference to treatment satisfaction, what is the impact of a switch to digital media for mandala art therapy for clients? 


\section{Method}

\section{Design}

This study employed an experimental, non-concurrent multiple baseline single subject research design (Watson \& Workman, 1981). The conventional practice in single subject design research involves the use of a minimum of three data points for a baseline phase in order to establish baseline stability (Byiers, Reichle, \& Symons, 2012). However, in this study one of the three baseline phases contained just two points. This variance was used given both the brevity applied setting of the intervention. The multiple baseline design was used in this study to examine the impact of a creating a mandala using digital media over a creating a mandala using conventional media across three behaviors. The independent variable in Phase A was the TAU, while the independent variable in Phase $\mathrm{B}$ was the TAU with digital media. The three dependent variables were GAD, vicarious trauma, and client satisfaction.

\section{Participants}

\section{Recruitment and Selection Criteria}

Data was collected from three pre-service art therapists at a private university in the Western US. All three participants reported experiencing vicarious traumatization because of direct client contact and volunteered. Participants were given a pre-screening packet that contained a demographic questionnaire and the IES-R. Potential participants who demonstrated a significant level of vicarious trauma related distress as indicated by an average total score of 24 or higher on the IES-R were considered for inclusion in this study. A score of greater than 24 indicates that PTSD is a clinical concern, and also indicates a high level of vicarious traumatization in a variety of populations (Devilly, Wright, \& Varker, 2009; Kadambi \& Truscott, 2007). Potential participants who were taking psychotropic medications, who had participated in mental health counseling related to the traumatic event, or who were participating in ongoing mental health counseling were excluded from this study. The participants were assigned pseudonyms to preserve confidentiality.

\section{Delores}

This participant identified as female, and as both Latino and Caucasian. Delores was in her final quarter of her Master's program, and reported using touch screen technology for six years. As a student of art therapy, Delores was very familiar with techniques and practices of art therapy. She scored 36 on the pre-screen IES-R, indicating a probable diagnosis of PTSD and therefore met inclusion criteria. Delores was in the process of completing her internship at an inpatient psychiatric ward for adults with severe and persistent mental illness. Delores worked closely with the patients in an art therapy setting, and heard many of their personal life histories which included multiple traumatic events. Delores reported experiencing symptoms of vicarious trauma 
including waves of emotion, avoidance of thought and emotion, and a feeling of numbness.

\section{Georgia}

This participant identified as a Caucasian female. Georgia had completed her coursework and internship in her Master's program, and was working toward the completion of her capstone project. She reported using touchscreen technology for three years. As a student of art therapy, Georgia was familiar with techniques and practices of art therapy. She scored 55 on the pre-screen IES-R, indicating a probable diagnosis of PTSD and therefore met inclusion criteria. Georgia completed her internship that involved providing art therapy services in a community mental health program, which provided grief and loss counseling to clients who had experienced death in their families. Georgia reported symptoms of vicarious trauma including feeling numb, making great effort to avoid thinking of her clients' stories, feeling irritable and angry, and having vivid dreams about her clients.

\section{Martha}

This participant identified as a Caucasian female. Martha was in the second quarter of her internship at a community mental health facility, which provided mental health services to children and families. She reported using touch screen technology for five years. As a student of art therapy, Martha was very familiar with techniques and practices of art therapy. Martha scored 27 on the pre-screen IES-R, indicating that PTSD is a clinical concern, and therefore met inclusion criteria. She reported symptoms of vicarious trauma including trouble falling and staying asleep, somatic reactions (such as sweating and heavy breathing) when recalling her clients' stories, and irritability.

\section{Measures}

\section{Demographic Survey}

This survey queried about the following variables: (a) age (b) gender, (c) education level, (d) race/ethnicity, and (e) years of experience using touch screen technology.

\section{Technology Self-Efficacy Scale (TSES)}

Participants' self-efficacy regarding the use of touch screen technology was assessed via the TSES. This measure is a 10-item rating scale that uses a 10-point Guttman scale $(1=$ not at all confident to $10=$ totally confident). The TSES was adapted from Compeau and Higgins' Computer-Self Efficacy Scale (CSES) touch screen technology. The CSES was intended to assess a person's confidence in their ability to use unfamiliar technology under a variety of conditions, such as if they have a user's manual at their disposal, if someone demonstrated proper use of the technology, or if they had 
used similar technology in the past (Compeau \& Higgins, 1995).

National Stressful Events Survey PTSD Short Scale: Severity of Posttraumatic Stress Symptoms-Adult (NSESSS-SA)

The NSESSS-SA is a measure used by both clinicians and researchers. It is intended for use in the initial assessment and to measure ongoing progress in the treatment of PSTD. The NSESSS-SA specifically measures the severity of PTSD symptoms. The scale has high internal consistency and convergent validity (LeBeau et al., 2014). Scores range from 0-to-36 with higher scores indicating greater severity of PTSD.

Impact of Events Scale - Revised (IES-R) Full Scale

The IES- $\mathrm{R}$ is a 22 item rating scale that assesses for subjective stress caused by traumatic events and has been used to measure vicarious traumatization (VT) in a variety of populations (Devilly, Wright, \& Varker, 2009; Kadambi \& Truscott, 2007). It is the most widely used self-port assessment for symptoms of trauma currently in use.

Participants are asked to indicate how frequently each of the 22 symptoms has occurred over the previous week. Total scores range from 0 -to-40, with higher scores indicating a greater severity of VT. The IES-R has good reliability, as well as concurrent and discriminative validity (Beck et al., 2008).

Impact of Events Scale - Revised (IES-R) Revised

Of the 22 items on the IES-R, 8 measure symptoms of avoidance. These 8 items were used to measure the symptoms of avoidance in participants. The Diagnostic and Statistical Manual of Mental Disorders ( $5^{\text {th }}$ ed.; DSM-V; American Psychiatric Association [APA], 2013) lists symptoms of avoidance under Criterion $\mathrm{C}$ in the diagnosis of PTSD and includes trauma-related thoughts, feelings, or reminders of the traumatic event. The avoidance subscale has been shown to have high internal consistency and test-retest reliability (Beck et al., 2008). Therefore, the symptoms of avoidance are a strong indicator of vicarious traumatization in mental health practitioners who work with traumatized clients (McLean \& Wade, 2003).

\section{Generalized Anxiety Disorder 7-item scale (GAD-7)}

The GAD-7 is a seven-item rating scale that assesses for anxiety. Participants are asked to report how often have certain seven indicators of anxiety impacted their lives over the past two weeks. A score of $0,1,2$, or 3 is assigned to the response categories of 'not at all', 'several days', 'more than half the days', and 'nearly every day', respectively. The scores of each of the seven items are then added together. A score of 5 or below indicates mild symptoms of anxiety, a score of 6-10 indicates moderate symptoms of anxiety, and a score of 15-18 indicates symptoms of severe anxiety. The GAD has good reliability, as well as criterion, construct, factorial, and procedural validity 
(Spitzer, Kroenke, Wiliams, \& Lowe, 2006).

Client Satisfaction Questionnaire 3 (CSQ-3)

The CSQ-3 is a short form of the Client Satisfaction Questionnaire. This short form contains three item areas on a 4-point, fully anchored Likert scale. Thus, a total score can range from 3 to 12 . Level of client satisfaction increases with numeric value. The items query client satisfaction with the therapeutic process (Larsen, Attkisson, Hargreaves, \& Nguyen, 1979). The CSQ-3 possesses excellent internal consistency (Creamer, Bell, \& Failla, 2003). In this particular study, the wordings of two items were modified to fit an art therapy intervention.

\section{Intervention}

\section{Baseline (Phase A-TAU)}

During the Baseline Period (BP), participants met with the researcher for weekly, approximately, one-hour sessions. A modified version of Machioldi's (2003) mandala directive was delivered. Participants were then provided with markers (Prismacolor Premier double-ended brush tip markers, 12 color set), oil pastels (Cray-Pas Expressionist, 12 color set), colored pencils (Prismacolor Premier, soft core, 24 color set), graphite pencils (Derwent graphic drawing pencils, 12 count, 6B-4H), a set of erasers (Prismacolor Premier kneaded, artgum, and plastic eraser set), a ruler (Breman Precision stainless steel cork back ruler, 15"), and an 8"x11" sheet of paper with a circle approximately six inches in diameter printed in the center. Participants were instructed to use the materials to fill in the shape however they wished. Participants drew for one half hour during each session.

\section{Intervention (Phase B-Electronic Media)}

During the Electronic Media Period (EMP), participants were provided with an iPad brand digital media tablet with the Art Set Pro app installed and a stylus. Art Set Pro closely replicates drawing and painting with the conventional materials used by participants in the Baseline Period (BP). During the first session of the EMP, the participants received brief instruction on the use of the Art Set Pro app with the iPad and stylus. Lastly, the participants were then given one half hour to create a mandala of their own.

\section{Therapist}

The art therapist was the first author (ED). She is a Licensed Mental Health Counselor (WA) and nationally Board Certified Art Therapist (ATR-BC). She holds master's degree in art therapy and was a doctoral student in counselor education. She has provided art therapy in multiple settings with clients having a wide variety of psychiatric diagnoses. 


\section{Apparatus}

\section{IPad}

The Apple iPad Air 2 tablet computer with a touchscreen interface. The iPad measures 0.24 " x 6.60" x 9.40" and weighs about 445.088 grams. Using the iPad, participants can create digital art via an installed application ("app"). Participants may choose to use a stylus to interact with an app, or they may use their fingers. The iPad allows participants to use tools that closely mimic conventional art media to create images of their choosing.

\section{App}

The app used in this study was the Art Set Pro - Edition app (version 1.2), which was released on February $28^{\text {th }}, 2015$. The app provides the opportunity to use digital versions of a wide variety of conventional art media, including mechanical, graphite and colored pencils, charcoal, paint markers, artist's markers, chalk and oil pastels, crayons, and oil paint. Users can choose to work on 28 different simulations of paper, which range in color, variety, and texture.

\section{Stylus}

Participants were given the option of using the Pencil by FiftyThree Digital Stylus, model number 53PW06 to create their images. Using the stylus, participants were able to achieve more precision in creating lines than is possible by just using a finger to draw or paint with. Bluetooth technology linked the stylus to the iPad, which permitted users to rest their palms on the screen of the iPad without unintentionally creating new marks on their artwork. This is a unique feature of digital art media, and is not possible with conventional media.

\section{Procedures}

This research project was reviewed and approved by the institutional review boards at Oregon State University and Antioch University-Seattle (OSU IRB Study \#7165). Participants were pre-screened for trauma symptom severity. Participants who self-reported experiencing one or more traumatic events and who received a score of 24 or higher on the IES-R and 1 or higher on the NSESSS were considered for inclusion in this study. Participants who were taking psychotropic medications or who were participating in ongoing mental health counseling were excluded from this study. As participants were current art therapy students at the institution that the researcher taught at throughout the duration of the study, any students who were in a class taught by the researcher at the time of the study or who might have been required to take a class taught by this researcher in the future were also excluded from this study. 
Three participants met aforenoted criteria for inclusion in the study. Following Watson and Workman's (1981) procedures for a nonconcurrent design, participants were assigned randomly to one of the three baseline conditions. Participants engaged in eight weekly individual sessions. At the beginning of the first session, written informed consent was obtained and the demographic questionnaire was administered. At the conclusion of the baseline phase, digital media was introduced in the form of an iPad and a stylus. The iPad was loaded with the Paper 53 app, and a circle approximately 5 " in diameter was pre-drawn on the screen. The participants were instructed to use the stylus to fill in the shape however they wished. They then drew for 30 minutes.

At the conclusion of each weekly intervention, the modified IES-R Avoidance Subscale, the GAD-7, and the CSQ-3 were administered. The IES-R was administered to assess for symptoms of vicarious trauma. The GAD-7 was administered to assess for symptoms of anxiety. The CSQ-3 was administered to assess each participant's level of satisfaction with the intervention.

Storage of the conventional art products followed standard procedures for such items (i.e., locked filing cabinet located in the locked office). The digital media art products required a different storage process. These items remained on the iPad that was password protected. It is critical to note cloud syncing capability of electronic tablets. This iPad's syncing mechanism was turned off. When not in use, the iPad itself was deposited in the same location as the conventional art products.

\section{Procedural Fidelity}

Procedural fidelity was determined via a 15-item checklist. Each item had a bimodal format (i.e., present/not present). The therapist completed this checklist for each session retrospectively at the end of the study. Treatment fidelity was calculated at $100 \%$. The checklist is available from the first author.

\section{Data Analysis}

Data was assessed using both visual analysis and statistical analysis (Spriggs \& Gast, 2010; Parker \& Vannest, 2009). To aid visual analysis, mean phase lines were plotted (Bulté \& Onghena, 2012). The graphs used for visual analysis were produced by Excel (Dixon et al., 2009). Also, the present study employed the nonoverlap of all pairs (NAP) as the statistical procedure (Parker \& Vannest, 2009). According to Parker, Vannest, Davis, \& Sauber, 2011) NAP is defined as "the percentage of all pairwise comparisons across Phases A and B, which show improvement across phases" (p. 312). There are four steps that are involved in the calculation of this percentage. These are: (a), the total number for pairs in calculated $\left(N_{\mathrm{a}} \mathrm{X} N_{\mathrm{b}}\right),(\mathrm{b})$ overlapping pairs are scored as 1 , tied pairs as .5, nonoverlapping pairs as 0 , and a total score is calculated, (c) this total score is subtracted from the total number of pairs, and (d) the resultant difference from this subtraction is divided from the total number of pairs. The NAP possesses good statistical power compared to other nonoverlap analysis procedures (Parker, Vannest, 
Davis, \& Sauber, 2011). Moreover, the NAP scores for each participant were determined by use of an online calculator designed for this analysis (Vannest, Parker, Gonen, \& Adiguzel 2016).

\section{Results}

\section{Vicarious Trauma Symptoms}

Figure 1 contains a graphical representation for the results for VT symptoms. Visual inspection of the Phase A median line and the Phase B median line revealed that two participants improved on VT symptoms as a result of the intervention. The VT symptoms for one participant remained stable across the phases. In terms of statistical analysis, medium to strong effect sizes were encountered with the NAP scores for two of three participants. The NAP score for Participant 1 was .75, for Participant 2 was .98 . Participant 3 had a weak effect size (NAP $=.42)$. In summary, there was no evidence that the switch to digital media had a negative effect on VT symptoms.

\section{GAD Symptoms}

Figure 2 contains a graphical representation of the results for GAD symptoms. Visual inspection of the Phase A median line and the Phase B median line revealed that two participants improved on GAD symptom as a result of the intervention. The GAD symptoms for one participant remained stable across the phases. In terms of statistical analysis, medium effect sizes were encountered with the NAP scores for two participants. The effect size found for Participant 1 was .92; the effect size found for Participant 2 was also .92; and the effect size found for Participant 3 was weak (NAP =.38). In summary, there was no evidence that the switch to digital media had a negative effect on GAD symptoms.

\section{Client Satisfaction}

Figure 3 contains a graphical representation of the results for client satisfaction. Phase A median line and the Phase B median line visual inspection showed that lient satisfaction did not change with the switch to digital media. Weak effect sizes were encountered with the NAP scores for all the three participants. The effect size found for Participant 1 was .21; the effect size found for Participant 2 was .63; while the effect size found for Participant 3 was .58. In conclusion, the switch to digital media had no effect upon client satisfaction. The average CSQ-3 scores for both conventional media $(\bar{x}=$ 10.28) and digital media $(\bar{x}=10.1)$ were high.

\section{Discussion}

This study endeavored to answer three research questions. Specifically, does a switch to digital media impact the following: (1) symptoms of GAD, (2) vicarious trauma, and (3) client satisfaction. The results suggest that a switch to digital media 
from conventional media had a positive effect in reduction of symptoms of anxiety and vicarious trauma, and had little effect in terms of client satisfaction.

Looking first at symptoms of GAD, two participants experienced reduced symptoms of anxiety over the course of the eight-week study. The third participant experienced a very slight reduction in symptoms. The findings suggest that the use of digital media in art therapy may be as effective as the use of conventional media in reducing symptoms. The data shows that the introduction of digital media did not have any negative effect on symptoms of generalized anxiety for any of the participants. It has been indicated that creating mandalas with conventional media results in reduced symptoms of anxiety according to Curry and Kasser (2005). However, the results of this study show that the use of digital media is no less effective than the use of conventional media in art therapy. One alternate explanation for this result is that the study occurred during an academic quarter, and all the participants were students at the same university. At this institution, the majority of a student's academic responsibility is satisfied two to three weeks before the end of the quarter. In general, students' anxiety levels are lower at the end of the quarter than at the beginning. Also, it could be reasoned that this timing contributed somewhat to the reduction in symptoms of anxiety.

Next, looking at symptoms of vicarious trauma, all three participants showed very little change in symptoms. The findings suggest that the introduction of digital media in art therapy had no negative impact on treatment of symptoms of vicarious trauma. Henderson, Rosen, and Mascaro (2007) showed that mandala-making with conventional media can reduce symptoms of PTSD. The results of this study demonstrate that the use of digital media in art therapy is no less effective that the use of conventional media in treating symptoms of PTSD. An alternate explanation for these results may be that all participants were continually exposed to trauma throughout the study and beyond its conclusion. Moreover, it could be argued that symptoms of vicarious trauma changed very little due to this sustained exposure to trauma.

The introduction of digital media had no effect on client satisfaction. Whether they used conventional media or digital media, participants reported high satisfaction with the their experience of therapy. An alternative explanation for this exists. It could be explained that a ceiling effect may have been at play. The highest possible score on the CSQ-3 is 12 and the average score across both conditions was 10.17, which is quite high. Finally, it could be reasoned that an assessment with a higher possible score may have yielded different results.

There exist five potential limitations to this study that should be considered. Two of these threats are related to internal validity--history, and instrumentation (Christ, 2007). First, each of the three participants were students in an art therapy program at the time of the study. The final sessions took place during the conclusion of the academic quarter, when students at this institution are commonly under less stress than during the rest of the quarter. Second, as aforenoted, a non-standard two-point baseline was used for the first participant in this study. This baseline length may have 
resulted in a slight lack of baseline information for this participant. Finally, though a procedural fidelity checklist was created and used, this checklist was done after the conclusion of the study and by the researcher. To ensure higher procedural fidelity, a checklist could have been created before the study commenced and used by a nonparticipant observer.

Three potential limitations to external validity which may have influenced the outcomes of the study. These were multiple treatment effect, experimenter effect, and novelty (Bracht \& Glass, 1968). First, a multiple treatment effect should be considered. Digital media provides a different tactile experience for participants. Independent from the switch of media, it is possible that this different tactile experience could have itself could have had an effect. Hinz (2009) commented that there may be a therapeutic benefit for some to use of a more structured and contained media such as digital one.

An experimenter effect may have influenced the outcomes of this study. The researcher administered the therapy was a former instructor of the participants. The participants may have experienced treatment differently if they were not previously known the researcher. The third potential limitation was novelty. The participants may have had greater interest in the new media and potentially shaped their answers.

Some art therapists may see the different tactile experience provided by the iPad and stylus as less therapeutic than that provided by conventional media. However, clients benefit from a variety of media. While the smooth and two-dimensional sensory experience offered by an iPad and stylus may not be a suitable media choice for every client, some clients may benefit from the use of more structured and controlled media (Hinz, 2009). Looking at the outcomes of this study, there appears to be no negative impact on client satisfaction or on the efficacy of the intervention as the result of the tactile experience provided by the digital media. As with all single subject design studies, replication of this experiment is needed to confirm the findings.

Rogers' (2003) theory on the diffusion of technology theory can assist understanding the implications that emerge from the present study. In particular, more art therapists must incorporate the use of digital media into their practice if the field is going to be spurred to greater adoption of this modality. When art therapists see new technology as an enhancement, they are more likely to use that technology in their work (Peterson, 2010). Digital art media possesses three specific benefits when compared to conventional media. First, digital media apparatuses such as an iPad and stylus take up less space than the wide assortment of tools essential for conventional art creation. While conventional art products require ample storage space, the art product of digital media takes up essentially no physical space. This efficiently solves the issue of storage of client art work, which is a difficulty faced that many practicing art therapists regularly grapple with. Second, many art therapists travel to a variety of settings to practice. The use of digital media may prove to be more convenient for traveling art therapists, as digital media tools are more portable than conventional media tools. Third, while 
conventional media tools require post-session cleaning time, digital media does not impose this activity.

The present article described an experimental study examining if a switch to digital media would have a deleterious impact on an art therapy intervention. The results suggested that such a switch does not. These results suggest that art therapist should begin to reconsider any blanket prejudices they possess against the employment of digital media. Replication of the present study is needed to further build the nomothetical net is the area of media selection in art therapy.

\section{References}

Adams, S. A., \& Riggs, S. A. (2008). An exploratory study of vicarious trauma among therapist trainees. Training and Education in Professional Psychology, 2(1), 2634. http:// doi.org/ 10.1037/1931-3918.2.1.26

American Art Therapy Association (2011). American Art Therapy Association Technology Committee [Brochure]. http://arttherapytech.files.wordpress.com/2011/01/aatatech_blog_brochure2.pdf

American Psychiatric Association. (2013). Diagnostic and statistical manual of mental disorders (5th). American Psychiatric Publishing.

Beck, J. G., Grant, D. M., Read, J. P., Clapp, J. D., Coffey, S. F., Miller, L. M., \& Palyo, S. A. (2008, August 11). The Impact of Event Scale -Revised:

Psychometric properties in a sample of motor vehicle accident survivors. Journal of Anxiety Disorders, 22(2), 187-198.

http://dx.doi.org/10.1016/j.janxdis.2007.02.007

Bracht, G. H., \& Glass, G. V. (1968). The external validity of experiments. American Educational Research Journal, 5, 437474. https://doi.org/10.3102/00028312005004437

Briere, J. (1995). Trauma symptom inventory: Professional manual [Measurement instrument]. http://www4.parinc.com

Bulté, I., \& Onghena, P. (2012). When the truth hits you between the eyes. A software tool for the visual analysis of single-case experimental data. Methodology, 8, 104114. doi:10.1027/1614-2241/a000042

Byiers, B. J., Reichle, J., \& Symons, F. J. (2012). Single-subject experimental design for evidence-based practice. American Journal of Speech-Language Pathology, 21(4), 397-414. http://doi.org/10.1044/1058-0360(2012/11-0036)

Carlton, N. R. (2014, February). Digital culture and art therapy. The Arts in Psychotherapy, 41, 41-45. http://doi.org/10.1016/j.aip.2013.11.006

Christ, T. J. (2007). Experimental control and threats to internal validity of concurrent and nonconcurrent multiple baseline designs. Psychology in the Schools, 44, 451459. http://doi.org/10.1002/pits.20237

Compeau, D. R., \& Higgins, C. A. (1995). Computer self-efficacy: Development of a measure and initial test. MIS Quarterly, 189-211. 
Creamer, M., Bell, R., \& Failla, S. (2003, December). Psychometric properties of the Impact of Event Scale-Revised. Behaviour Research and Therapy, 41(12), 14891496. http:// doi.org/2003.07.010

Curry, N. A., \& Kasser, T. (2005). Can coloring mandalas reduce anxiety? Art Therapy: Journal of the American Art Therapy Association, 22(2), 81-85. http:// doi.org/10.1080/07421656.2005.10129441

Derogatis, L. R. (1994). Symptom Checklist-90-Revised [Measurement instrument]. Published instrument. http://www.pearsonclinical.com

Devilly, G. J., Wright, R., \& Varker, T. (2009). Vicarious trauma, secondary traumatic stress or simply burnout? Effect of trauma therapy on mental health professionals. Australian and New Zealand Journal of Psychiatry, 43(4), 373-385. http://doi.org/10.1080/00048670902721079

Ferszt, G. G., Hayes, P. M., Defedele, S., \& Horn, L. (2004). Art therapy with incercerated women who have experienced the death of a loved one. Art Therapy: Journal of the American Art Therapy Association, 21, 191-199.

Ferszt, G. G., Hays, P. M., DeFedele, S., \& Horn, L. (2004). Art therapy with incarcerated women who have experienced the death of a loved one. Art Therapy, 21(4), 191-199. http://doi.org/10.1080/07421656.2004.10129695

Foa, E. B. (1998). PDS. Posttraumatic Stress Diagnostic Scale [Manual]. www.psychcorp.com

Gersch, I., \& Sao Joao Goncalves, S. (2010, December 21). Creative arts therapies and educational psychology: Let's get together. International Journal of Art Therapy, 1(1), 22-32. http:// doi.org/dx.doi.org/10.1080/17454830600674050

Goolkasian, P. (n.d.). Spielberger's State Anxiety Inventory [Measurement instrument]. http://www.mindgarden.com/products/staisad.htm

Henderson, P., Rosen, D., \& Mascaro, N. (2007). Empirical study on the healing nature of mandalas. Psychology of Aesthetics, Creativity, and the Arts, 1(3), 148-154.

Hinz, L. D. (2009). Expressive therapies continuum: A framework for using art in therapy. Routledge.

Kadambi, M. A., \& Truscott, D. (2007). Vicarious trauma among therapists working with sexual violence, cancer and general practice. Canadian Journal of Counselling and Psychotherapy, 38(4). http:// doi.org/10.1080/87568225.2010.486296

Kapitan, L. (2011). Introduction to the special issue on art therapy's response to technodigital culture. Art Therapy: Journal of the American Art Therapy Association, 26, 50-51.

Kapitan, L. (Ed.). (2009). Special issue: special issue on art therapy's response to technodigital culture [Special issue]. Art Therapy: Journal of the American Art Therapy Association, 26(2).

Larsen, D. L., Attkisson, C. C., Hargreaves, W. A., \& Nguyen, T. D. (1979). Assessment of client/patient satisfaction: development of a general scale. Evaluation and Program Planning, 2, 197-207. http://doi.org/10.1016/0149-7189(79)90094-6

LeBeau, R., Mischel, E., Resnick, H., Kilpatrick, D., Friedman, M., \& Craske, M. (2014, August 15). Dimensional assessment of posttraumatic stress disorder in DSM-5. Psychiatry Research, 218(1-2), 143-147. http://doi.org/10.1016/j.psychres.2014.03.032 
McLean, S., \& Wade, T. D. (2003). The contribution of therapist beliefs to psychological distress in therapists: An investigation of vicarious traumatization, burnout, and symptoms of avoidance and intrusion. Behavioral and Cognitive Psychotherapy, 31, 417-728. http://doi.org/10.1017/S135246580300403X

Nowicka-Sauer, K. (2007). Patients' perspective: lupus in patients' drawings. Clinical Rheumatology, 29. http://doi.org/10.1007/s10067-007-0619-9

Orr, P. P. (2009). Technology training for future art therapists: Is therea need? Art Therapy: Journal of the American Art Therapy Association, 23(4), 191-196. http://doi.org/10.1080/07421656.2006.10129329

Parker, R. I., \& Vannest, K. (2009). An improved effect size for single-case research: Nonoverlap of all pairs. Behavior Therapy, 40(4), 357-367. http://doi.org/10.1016/j.beth.2008.10.006

Parker, R. I., Vannest, K. J., Davis, J. L., \& Sauber, S. B. (2011). Combining nonoverlap and trend for single-case research: Tau-U. Behavior Therapy, 42(2), 284-299. http://doi.org/10.1016/j.beth.2010.08.006

Pearlman, L. A., \& Mac Ian, P. S. (1995). Vicarious traumatization: An Empirical Study of the effects of trauma work on trauma therapists. Professional Psychology: Research and Practice, 26, 558-565.

Peterson, B. C. (2010). The media adoption stage model of technology for art therapy. Art Therapy: Journal of the American Art Therapy Association, 27(1), 26-31. http://doi.org/10.1080/07421656.2010.10129565

Pew Research Center. (2015). Technology device ownership: 2015. http://www.pewinternet.org/2015/10/29/technology-device-ownership-2015/

Pew Research Center. (2017). Internet/broadband fact sheet. Washington, DC: Author. http://www.pewinternet.org/fact-sheet/internet-broadband/

Rogers, E. M. (2003). Diffusion of innovations. Simon and Schuster.

Slegelis, M. H. (1987). A study of Jung's mandala and its relationship to art psychotherapy. The Arts in Psychotherapy, 14(4), 301-311. http://doi.org/10.1016/0197-4556(87)90018-9

Spitzer, R. L., Kroenke, K., Wiliams, J. B., \& Lowe, B. (2006, May 22). A brief measure for assessing generalized anxiety disorder: The GAD-7. Archives of Internal Medicine, 166(10). www.patient.co.uk

Spriggs, A. D., \& Gast, D. L. (2010). Visual representation of data. Single Subject Research Methodology in Behavioral Sciences, 166-198.

Vannest, K. J., Parker, R. I., \& Gonen, O. (2011). Single Case Research: Web based calculators for SCR analysis. Texas A\&M University. http://doi.org/10.1080/17454830600674050

Watson, P. J., \& Workman, E. A. (1981). The non-concurrent multiple baseline acrossindividuals design: An extension of the traditional multiple baseline design. Journal of Behavior Therapy and Experimental Psychiatry, 12(3), 257-259. http://doi.org/10.1016/0005-7916(81)90055-0

Wilson, J. P., \& Lindy, J. D. (1994). Countertransference in the treatment of PTSD. New Guilford Press.

Wolf, R. I. (2007). Advances in phototherapy training. The Arts in Psychotherapy, 34(2), 124-133. http://doi.org/10.1016/j.aip.2006.11.004 
Figure 1

Symptoms of Vicarious Trauma 


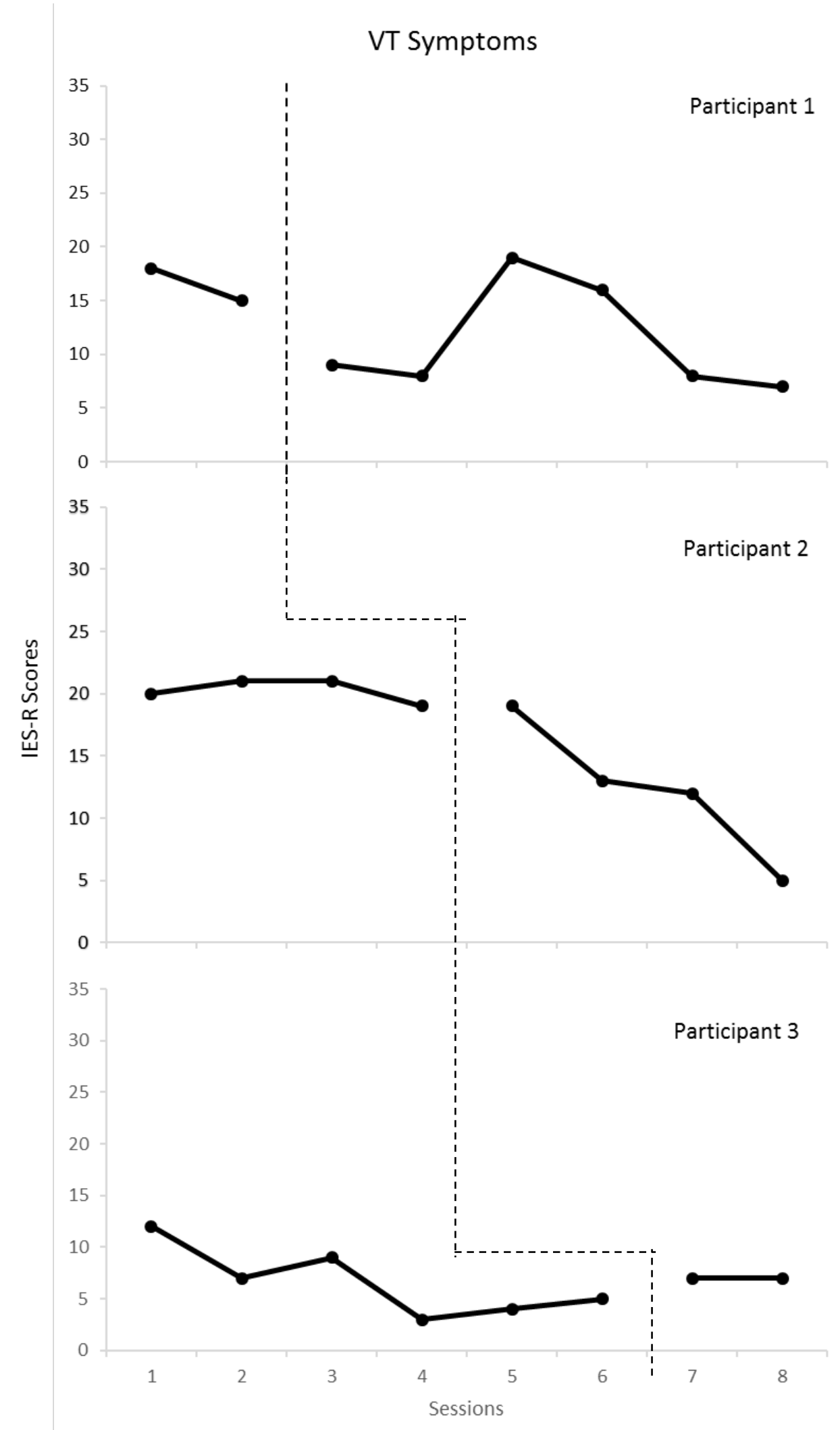


Figure 2

Symptoms of Generalized Anxiety 


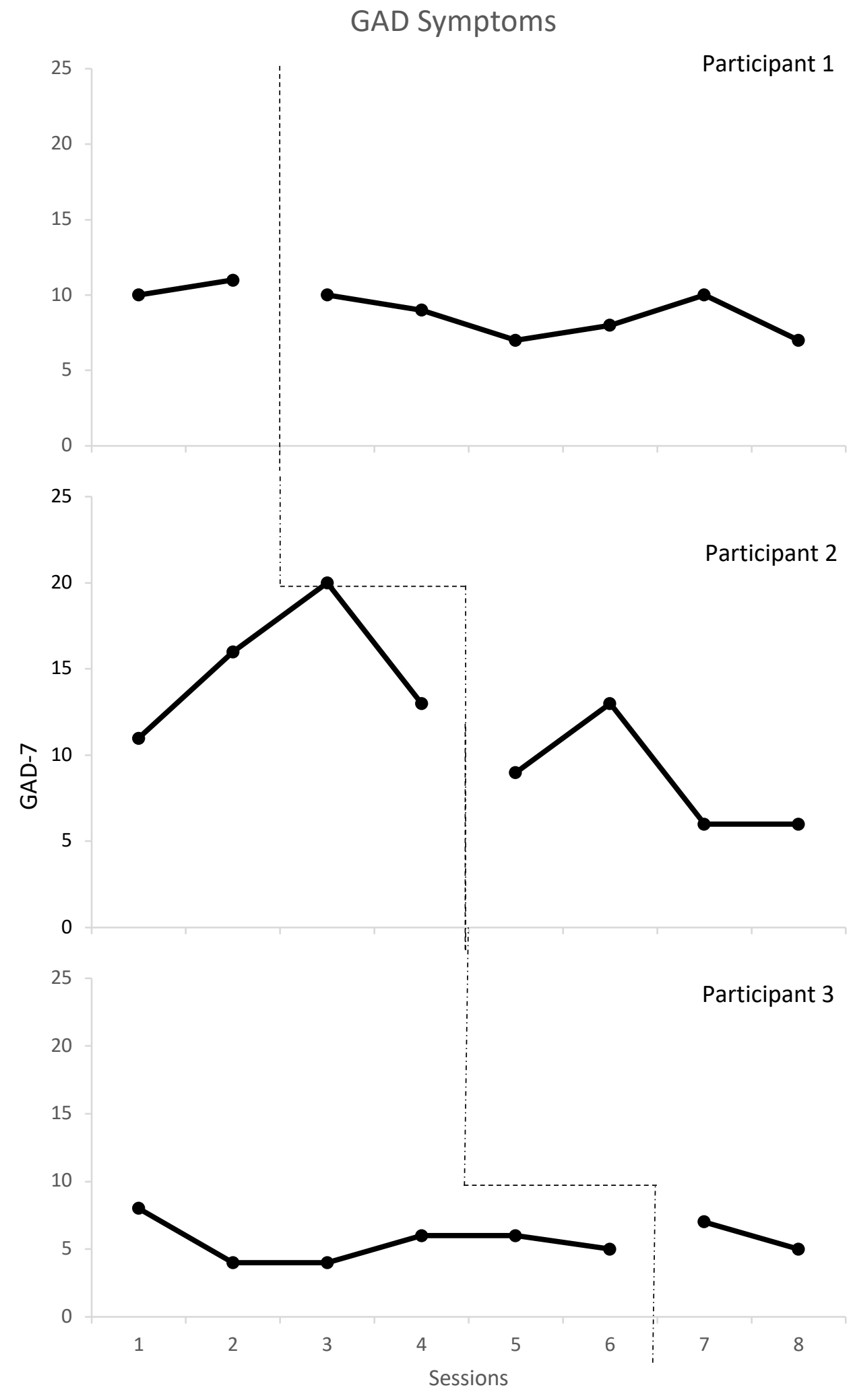


Figure 3

Client Satisfaction 


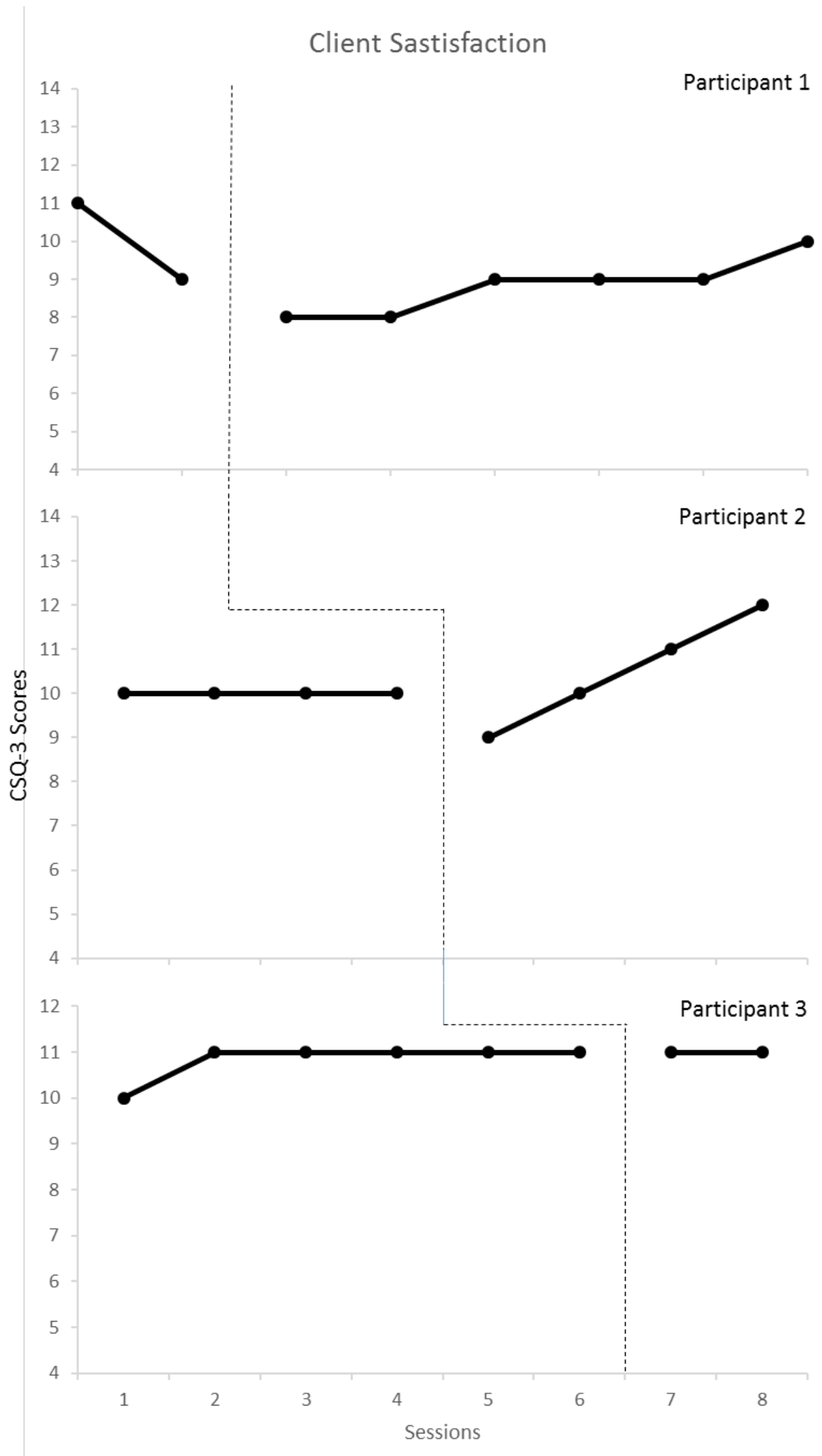

